\title{
COMPUTABILITY BY PROBABILISTIC TURING MACHINES
}

\author{
BY
}

\author{
EUGENE S. SANTOS
}

\begin{abstract}
In the present paper, the definition of probabilistic Turing machines is extended to allow the introduction of relative computability. Relative computable functions, predicates and sets are discussed and their operations investigated. It is shown that, despite the fact that randomness is involved, most of the conventional results hold in the probabilistic case. Various classes of ordinary functions characterizable by computable random functions are introduced, and their relations are examined. Perhaps somewhat unexpectedly, it is shown that, in some sense, probabilistic Turing machines are capable of computing any given function. Finally, a necessary and sufficient condition for an ordinary function to be partially recursive is established via computable probabilistic Turing machines.
\end{abstract}

I. Introduction. In a well-known paper [11], A. M. Turing defined a class of computing machines now known as Turing machines. These machines may be used to characterize a class of functions known as the partially recursive functions [3].

As it stands, Turing machines are deterministic machines. Since probabilistic machines have received wide interest in recent years [1], [8], it is natural to inquire about what will happen if random elements are allowed in a Turing machine. This has led the author to consider probabilistic Turing machines (PTM) in an earlier paper [9]. It turns out that, much like the Turing machines, PTM's may be used to characterize a class of random functions, the partially computable random functions.

In the present paper, the definition of PTM is extended to allow the introduction of relative computability. Relative computable functions, predicates and sets are discussed and their operations are investigated. It is shown that, despite the fact that randomness is involved, most of the conventional results hold in the probabilistic case.

The class of ordinary functions which are partially computable random functions is shown to be equivalent to the class of partially recursive functions. In this sense, we gain nothing by considering PTM's. However, it is shown that in some other

Presented to the Society, January 23, 1970 under the title On probabilistically computable functions; received by the editors September 7, 1970.

AMS 1970 subject classifications. Primary 02F15, 94A35; Secondary 94A30, $02 \mathrm{~F} 10$.

Key words and phrases. Probabilistic Turing machines, Turing machines, probabilistically computable functions, partially recursive functions, computable random functions, computable random sets, computable random predicates.

Copyright (C) 1971, American Mathematical Society 
sense we do gain something by considering PTM's. Mathematically speaking, this amounts to the fact that there exist classes of ordinary functions characterizable by PTM's which contain the class of partially recursive functions as proper subclass. One such class was given in [9]. Various other classes are discussed in the present paper and their relations are investigated. Perhaps somewhat unexpectedly, it is shown that, in some sense, PTM's are capable of computing any given function.

The paper concludes with some discussions on computable PTM's. A necessary and sufficient condition for an ordinary function to be partially recursive is established via computable PTM's.

II. Random sets, predicates and functions. In this section, we shall give a formal definition of random sets, predicates and functions and other related concepts which will be needed in later discussions. It is easily seen that they are generalizations of the conventional concepts. Moreover, they reduce to their counterparts in the conventional theory if the random elements are removed.

The symbols $\boldsymbol{X}$ and $\boldsymbol{Y}$ will stand for ordinary spaces of objects.

Definition 2.1. A random set $C$ in $X$ is characterized by the function $\mu_{C}$ from $\boldsymbol{X}$ into $[0,1]$. A $k$-ary random set in $\boldsymbol{X}$ is a random set in $\boldsymbol{X}^{k}=\boldsymbol{X} \times \boldsymbol{X} \times \cdots \times \boldsymbol{X}$ ( $k$ times).

REMARK. $\mu_{C}(x)$ is the probability that $x \in C$. If $\mu_{C}(x)=0$ or 1 for every $x \in X, C$ reduces to an ordinary set. In this case, we say that $C$ is a crisp set.

Definition 2.2. Let $C$ and $D$ be random sets in $X$. Then

1. $C=D$ iff (if and only if) $\mu_{C}=\mu_{D}$.

2. $C \subseteq D$ iff $\mu_{C} \leqq \mu_{D}$.

3. The complement of $C$ is the random set $\sim C$ where $\mu_{\sim C}=1-\mu_{C}$.

4. The intersection of $C$ and $D$ is the random set $C \cap D$ in $X$ where $\mu_{C \cap D}=\mu_{C} \cdot \mu_{D}$.

5. The union of $C$ and $D$ is the random set $C \cup D$ in $X$ where $\mu_{C \cup D}$ $=1-\left(1-\mu_{C}\right)\left(1-\mu_{D}\right)=\mu_{C}+\mu_{D}-\mu_{C} \cdot \mu_{D}$.

In the above definition, we suppress the argument of a function whenever an equality or inequality holds for all values of the argument are used. This convention will be used throughout the entire paper to simplify our notations.

It is clear that the operations of complementation, intersection and union for random sets obey most of the corresponding rules of ordinary set theory.

Definition 2.3. Let $C$ and $D$ be random sets in $X$ and $Y$, respectively. Then $C \times D$ is the random set in $\boldsymbol{X} \times \boldsymbol{Y}$ such that for every $x \in \boldsymbol{X}, y \in \boldsymbol{Y}$,

$$
\mu_{C \times D}(x, y)=\mu_{C}(x) \cdot \mu_{D}(y) .
$$

Definition 2.4. A random predicate $P$ in $\boldsymbol{X}$ is characterized by the function $\mu_{P}$ from $\boldsymbol{X}$ into $[0,1]$. A $k$-ary predicate in $\boldsymbol{X}$ is a random predicate in $\boldsymbol{X}^{k}$.

REMARK. $\mu_{P}(x)$ is the truth value of the statement $P(x)$, i.e., the probability that $P(x)$ is true. 
Definition 2.8. Let $P$ and $Q$ be random predicates in $X$. Then

1. $P=Q$ iff $\mu_{P}=\mu_{Q}$.

2. $\sim P$ (read "not $P$ ") is the random predicate in $X$ with $\mu_{\sim P}=1-\mu_{P}$.

3. $P \wedge Q$ (read " $P$ and $Q$ ") is the random predicate in $X$ with $\mu_{P \wedge Q}=\mu_{P} \cdot \mu_{Q}$.

4. $P \vee Q$ (read " $P$ or $Q$ ") is the random predicate in $X$ with $\mu_{P \vee Q}$ $=1-\left(1-\mu_{P}\right)\left(1-\mu_{Q}\right)$.

Definition 2.5. Let $P$ be a random predicate in $X$. The extension of $P$ is the random set $E_{P}$ where $\mu_{E_{P}}=\mu_{P}$.

Corollary 2.1. Let $P$ and $Q$ be random predicates in $X$. Then

1. $E_{\sim P}=\sim E_{P}$.

2. $E_{P \wedge Q}=E_{P} \cap E_{Q}$.

3. $E_{P \vee Q}=E_{P} \cup E_{Q}$.

4. $P=Q$ iff $E_{P}=E_{Q}$.

Definition 2.6. A random function $f$ from $X$ into $Y$ is characterized by the function $\mu_{f}$ from $\boldsymbol{X} \times \boldsymbol{Y}$ into $[0,1]$ where

$$
\sum_{y \in \boldsymbol{Y}} \mu_{f}(x, y) \leqq 1
$$

If, in (2.1), the equality holds for all $x \in X$, then $f$ is total. A $k$-ary random function in $\boldsymbol{X}$ is a random function from $\boldsymbol{X}^{k}$ into $\boldsymbol{X}$.

REMARK. $\mu_{f}(x, y)$ is the probability that $f(x)$ is equal to $y$. The inequality (2.1) allows one to consider functions which are undefined for some $x \in X$.

If the range of $\mu_{f}$ consists of only two numbers, 0 and $1, f$ reduces to an ordinary function. In this case, we say that $f$ is a crisp function and the conventional notations of ordinary functions will be used freely, e.g., $f(x)=y$ if $\mu_{f}(x, y)=1$, etc.

For ease of notation, we shall follow the suggestion of Scott [10] by introducing a new symbol $\Omega$ to stand for the "undefined". Thus, if $f$ is a random function from $\boldsymbol{X}$ into $\boldsymbol{Y}$, define

$$
\mu_{f}(x, \Omega)=1-\sum_{y \in Y} \mu_{f}(x, y)
$$

for all $x \in X$, i.e., $\mu_{f}(x, \Omega)$ is the probability that $f$ is undefined at $x$.

Definition 2.7. Two random functions $f$ and $g$ from $\boldsymbol{X}$ into $\boldsymbol{Y}$ are equivalent with threshold $\lambda, 0 \leqq \lambda<1$, iff for all $x \in X$,

$$
\sum_{y \in \boldsymbol{Y}^{\prime}} \mu_{f}(x, y) \cdot \mu_{g}(x, y)>\lambda
$$

where $\boldsymbol{Y}^{\prime}=\boldsymbol{Y} \cup\{\Omega\}$. In symbols, $f^{\lambda} \boldsymbol{\sim}$.

REMARK. $f^{\lambda} g$ means for every $x \in X$, the probability that $f(x)=g(x)$ is larger than $\lambda$. If $f$ is a crisp function, (2.2) reduces to $\mu_{g}(x, f(x))>\lambda$ for all $x \in X$. Here, we use the convention that $f(x)=\Omega$ if $f$ is undefined at $x$. 
Definition 2.8. Let $f$ and $g$ be random functions from $\boldsymbol{X}$ into $\boldsymbol{Y}$. Define

$$
m(f, g)=\prod_{x \in \boldsymbol{X}}\left\{\sum_{y \in \boldsymbol{Y}^{\prime}} \mu_{f}(x, y) \cdot \mu_{g}(x, y)\right\}
$$

where $\boldsymbol{Y}^{\prime}=\boldsymbol{Y} \cup\{\Omega\}$.

REMARK. $m(f, g)$ is the probability that for all $x \in X, f(x)=g(x)$. If $f$ is a crisp function, (2.3) reduces to

$$
m(f, g)=\prod_{x \in \boldsymbol{X}} \mu_{g}(x, f(x))
$$

Here, again, we use the convention that $f(x)=\Omega$ if $f$ is undefined at $x$.

REMARK. Infinite product in the present paper differs slightly from the widely accepted one in the sense that we allow convergent to 0 . Formally, let $\left\{a_{n}\right\}$ be a sequence of real numbers. We define

$$
\prod_{n=1}^{\infty} a_{n}=\lim _{N \rightarrow \infty} \prod_{n=1}^{N} a_{n}
$$

Since all sequences $\left\{a_{n}\right\}$ under consideration have the property $0 \leqq a_{n} \leqq 1$ for all $n$, $\prod a_{n}$ always converges.

\section{Probabilistic Turing machines.}

Definition 3.1. A probabilistic Turing machine (PTM) may be defined through the specification of three mutually disjoint finite nonempty sets $A, B$, and $S$; a function $p$ from $S \times U \times V \times S$ into [0, 1] where $U=A \cup B, V=U \cup S \cup\{+,-, \cdot\}$, ,,$+- \cdot \notin U \cup S$; and a function $h$ from $S$ into $[0,1]$. The functions $p$ and $h$ satisfy the following conditions:

1. $\sum_{v \in V} \sum_{s^{\prime} \in S} p\left(s, u, v, s^{\prime}\right)=1$ for every $s \in S, u \in U$, and

2. $\sum_{s \in S} h(s)=1$.

The sets $A$ and $B$ are, respectively, the printing and auxiliary alphabets. The set $S$ is the set of internal states. $h(s)$ is the probability that the initial state is $s$ and $p\left(s, u, v, s^{\prime}\right)$ gives the probability of the "next act" of the PTM given that its present state is $s$ and input $u$ is applied. The "next act" of a PTM is determined by $v$ and may be any one of the conventional Turing machine operations.

1. $v \in U$ : replace $u$ by $v$ on the scanned square and go to state $s^{\prime}$.

2. $v=+:$ move one square to the right and go to state $s^{\prime}$.

3. $v=-$ : move one square to the left and go to state $s^{\prime}$.

4. $v=\cdot$ : stop.

5. $v \in S$ : go to either $v$ or $s^{\prime}$ depending on a given random set.

The functions $p$ and $h$ will be referred to as the transition function and initial distribution, respectively. If $h$ is concentrated at a single state $s_{0} \in S$, i.e., $h\left(s_{0}\right)=1$ and $h(s)=0$ for $s \neq s_{0}$, then we say that $s_{0}$ is the initial state. 
Due to condition 1 of Definition 3.1, some "next act" is certain. Therefore, the transition function may be defined by giving only those values of $p\left(s, u, v, s^{\prime}\right)$ for which $v \neq \cdot$ and $p\left(s, u, v, s^{\prime}\right) \neq 0$. This simplifying scheme for the definition of $p$ will be used throughout the entire paper.

Definition 3.2. Let $Z=(A, B, S, p, h)$ be a PTM. Then

1. $Z$ is deterministic iff the range of both $p$ and $h$ consists of only two numbers, 0 and 1 .

2. $Z$ is simple iff $p\left(s, u, v, s^{\prime}\right)=0$ for every $s, s^{\prime} \in S, u \in A \cup B$, and $v \in S$.

Observe that the conventional Turing machines are deterministic PTM and the PTM introduced by the author in an earlier paper [9] are simple PTM according to the above definitions.

In the case of a deterministic PTM, the transition function $p$ is uniquely determined by the set $\mathscr{P}=\left\{\left(s, u, v, s^{\prime}\right): p\left(s, u, v, s^{\prime}\right)=1\right.$ and $\left.v \neq \cdot\right\}$.

Notation. Let $C$ be an ordinary set. The collection of all finite sequences of symbols of $C$ will be denoted by $C^{*}$. For convenience sake, we shall assume that $C^{*}$ contains $e$ where $e x=x=x e$ for all $x \in C^{*}$.

Definition 3.3. Let $Z=(A, B, S, p, h)$ be a PTM. Expressions of $Z$, tape expressions of $Z$ and words of $Z$ are, respectively, elements of $(A \cup B \cup S)^{*}$, $(A \cup B)^{*}$, and $A^{*}$.

In what follows, if $Z=(A, B, S, p, h)$ is a PTM, then we assume that $A$ contains the symbol 1 and $B$ contains the symbols * and $b$, where $b$ stands for blank.

Definition 3.4. Let $Z=(A, B, S, p, h)$ be a PTM. An expression $\alpha$ of $Z$ is an instantaneous description of $Z$ iff

1. $\alpha$ contains exactly one $s \in S$ and $s$ is not the rightmost symbol of $\alpha$,

2. the leftmost symbol of $\alpha$ is not $b$, and

3. the rightmost symbol of $\alpha$ is not $b$ unless it is the symbol immediately to the right of $s$.

The collection of all instantaneous descriptions of $Z$ will be denoted by $\mathscr{I}(Z)$. If $\alpha$ is an instantaneous description of $Z$ which contains $s \in S$ and $u$ is the symbol immediately to the right of $s$, then we say that $s$ is the state of $Z$ at $\alpha$ and $u$ the symbol scanned by $Z$ at $\alpha$.

The above definition differs slightly from that given in [9]. It does not allow initial and final occurrences of $b$ unless $b$ is the symbol scanned by the PTM at that instant. The advantages of the present definition will be apparent as we proceed.

Notation. Let $Z=(A, B, S, p, h)$ be a PTM.

1. If $\alpha$ is an expression of $Z$ and $n$ a positive integer, then $\alpha^{n}$ will denote the expression $\alpha \alpha \cdots \alpha$ ( $n$ times) that consists of $n$ occurrences of $\alpha$. For completeness sake, we take $\alpha^{0}=e$.

2. With each nonnegative integer $n$, we associate the tape expression $\bar{n}=1^{n}$.

3. If $\alpha$ is an expression of $Z$, then $\langle\alpha\rangle$ will denote the word of $Z$ obtained by striking out all symbols in $\alpha$ not belonging to $A$ if $\alpha$ contains symbols from $A$; otherwise $\langle\alpha\rangle=b$. 
4. Unless otherwise stated, the letters $w, x, y$, with or without subscripts, will represent words of $Z$. Moreover, we shall write

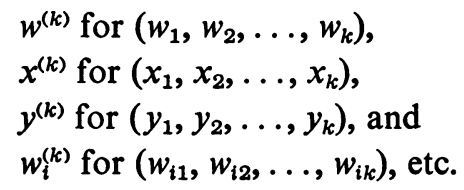

5. With each $k$-tuple $w^{(k)}$ of words of $Z$, we associate the tape expression

$$
\overline{w^{(k)}}=\overline{\left(w_{1}, w_{2}, \ldots, w_{k}\right)}=\left\langle w_{1}\right\rangle *\left\langle w_{2}\right\rangle * \cdots *\left\langle w_{k}\right\rangle \text {. }
$$

Observe that if $\alpha \in A^{*}$, then $\langle\alpha\rangle=\alpha$. Moreover, if $A=\{1\}$, then $\langle\alpha\rangle=\langle\bar{n}\rangle$ where $n$ is the number of occurrences of 1 in $\alpha$.

In Definitions 3.5 to 3.8 below, $Z=(A, B, S, p, h)$ is a PTM and $T$ a random set in $A^{*}$.

Definition 3.5. For every $\alpha, \beta \in \mathscr{I}(Z)$, define

$$
\begin{aligned}
& q_{z, T}(\alpha, \beta)=p\left(s, u, u^{\prime}, s^{\prime}\right) \quad \text { if } \alpha=\gamma s u \delta, \quad \beta=\gamma s^{\prime} u^{\prime} \delta, \quad u^{\prime} \neq u \text {; } \\
& =p\left(s, u,+, s^{\prime}\right) \text { if } \alpha=\gamma s u u^{\prime} \delta, \quad \beta=\gamma u s^{\prime} u^{\prime} \delta, \quad \gamma u \neq b, \\
& \text { or } \alpha=s^{\prime} u^{\prime} \delta, \quad \beta=s^{\prime} u^{\prime} \delta, \quad u=b, \\
& \text { or } \alpha=\gamma s u, \quad \beta=\gamma u s^{\prime} b, \quad \gamma u \neq b, \\
& \text { or } \alpha=s u, \quad \beta=s^{\prime} b, \quad u=b ; \\
& =p\left(s, u,-, s^{\prime}\right) \text { if } \alpha=\gamma u^{\prime} s u \delta, \quad \beta=\gamma s^{\prime} u^{\prime} u \delta, \quad u \delta \neq b \text {, } \\
& \text { or } \alpha=\gamma u^{\prime} s u, \quad \beta=\gamma s^{\prime} u^{\prime}, \quad u=b, \\
& \text { or } \alpha=s u \delta, \quad \beta=s^{\prime} b u \delta, \quad u \delta \neq b \text {, } \\
& \text { or } \alpha=s u, \quad \beta=s^{\prime} b, \quad u=b \text {; } \\
& =p\left(s, u, u, s^{\prime}\right)+\sum_{s^{\prime \prime} \in S} p\left(s, u, s^{\prime}, s^{\prime \prime}\right) \cdot \mu_{T}(\langle\alpha\rangle) \\
& +\sum_{s^{\prime \prime} \in S} p\left(s, u, s^{\prime \prime}, s^{\prime}\right) \cdot\left[1-\mu_{T}(\langle\alpha\rangle)\right] \\
& \text { if } \alpha=\gamma s u \delta, \quad \beta=\gamma s^{\prime} u \delta ; \\
& =0 \quad \text { otherwise; }
\end{aligned}
$$

where $\gamma, \delta \in(A \cup B)^{*}, s, s^{\prime} \in S$, and $u, u^{\prime} \in A \cup B$.

RemarK. $q_{z, T}(\alpha, \beta)$ is the probability that the "next" instantaneous description of $Z$ relative to $T$ will be $\beta$ given that $Z$ "starts" with instantaneous description $\alpha$.

The above definition is tailored in such a way that initial and final occurrences of $b$ are automatically removed.

Definition 3.6. For every $\alpha, \beta \in \mathscr{I}(Z)$ and $n=0,1,2, \ldots$, define inductively

$$
\begin{aligned}
q_{Z, T}^{(0)}(\alpha, \beta) & =1 \quad \text { if } \alpha=\beta, \\
& =0 \quad \text { if } \alpha \neq \beta ; \\
q_{Z, T}^{(n)}(\alpha, \beta) & =\sum_{\gamma \in \mathcal{F}(Z)} q_{Z, T}^{(n-1)}(\alpha, \gamma) q_{z, T}(\gamma, \beta) .
\end{aligned}
$$


REMARK. $q_{Z, T}^{(n)}$ is the probability that the instantaneous description of $Z$ relative to $T$ will be $\beta$ "after $n$ steps" given that $Z$ " starts" with $\alpha$.

By induction, one shows that for every $\alpha \in \mathscr{I}(Z)$ and $n \geqq 0$,

$$
\sum_{\beta \in \mathscr{S}(Z)} q_{Z, T}^{(n)}(\alpha, \beta) \leqq 1
$$

Definition 3.7. For every $\alpha, \beta \in \mathscr{I}(Z)$ and $n=1,2, \ldots$, define

$$
t_{Z, T}^{(n)}(\alpha, \beta)=p(s, u, \cdot, s) q_{Z, T}^{(n-1)}(\alpha, \beta)
$$

where $s$ is the state of $Z$ at $\beta$ and $u$ the symbol scanned by $Z$ at $\beta$. Moreover, define

$$
t_{z, T}(\alpha, \beta)=\sum_{n=1}^{\infty} t_{z, T}^{(n)}(\alpha, \beta) .
$$

REMARK. $t_{Z, T}^{(n)}(\alpha, \beta)$ is the probability that $Z$ will "terminate" with $\beta$ relative to $T$ "after $n$ steps" given that $Z$ "starts" with $\alpha . t_{z, T}(\alpha, \beta)$ is the probability that $Z$ will "terminate" with $\beta$ relative to $T$ "after a finite number of steps" given that $Z$ “starts" with $\alpha$.

That $t_{z, T}(\alpha, \beta)$ converges follows from the fact that for every $\alpha \in \mathscr{I}(Z)$ and $N \geqq 0$,

$$
\sum_{\beta \in \mathscr{\mathcal { F }}(Z)} q^{(N)}(\alpha, \beta)=\sum_{\beta \in \mathscr{\mathcal { F }}(Z)}\left[q^{(N+1)}(\alpha, \beta)+t^{(N+1)}(\alpha, \beta)\right]
$$

or

$$
\sum_{\beta \in \mathscr{F}^{(Z)}}\left[\sum_{n=1}^{N} t_{Z, T}^{(n)}(\alpha, \beta)+q_{Z, T}^{(N)}(\alpha, \beta)\right]=1 .
$$

It is interesting to note that with each PTM, we may associate a Markov chain whose states are the instantaneous descriptions of the PTM plus an additional absorbing state [2] corresponding to the termination of the PTM.

DEFINITION 3.8. For each positive integer $k$, we associate a $k$-ary random function $\Phi_{Z, T}^{(k)}$ in $A^{*}$ as follows:

$$
\mu_{\Phi_{Z, T}^{(k)}}\left(w^{(k)}, w\right)=\sum_{\beta \in \mathscr{T}(Z) ;\langle\beta\rangle=w} \sum_{s \in S} h(s) t_{z, T}\left(\overline{s w^{(k)}}, \beta\right)
$$

where $w^{(k)} \in\left(A^{*}\right)^{k}$ and $w \in A^{*}$. If $k=1$, we shall write $\Phi_{z, T}$ for $\Phi_{Z, T}^{(1)}$.

That $\Phi_{Z, T}^{(k)}$ is a random function follows immediately from the definition of $t_{Z, T}$.

It is clear from the above definitions that if $Z$ is simple, then, for every $k, \Phi_{Z, T_{1}}^{(k)}$ $=\Phi_{Z, T_{2}}^{(k)}$ for arbitrary random sets $T_{1}$ and $T_{2}$ in $A^{*}$. In this case, we shall write $\Phi_{Z}^{(k)}$ for $\Phi_{Z, T}^{(k)}$.

TheORem 3.1. Let $Z=(A, B, S, p, h)$ be a PTM. There exists a PTM $Z^{\prime}$ $=\left(A, B, S^{\prime}, p^{\prime}, h^{\prime}\right)$ where $h^{\prime}$ is concentrated at a single state, and for every random set $T$ in $A^{*}$ and $k=1,2, \ldots, \Phi_{Z, T}^{(k)}=\Phi_{Z^{\prime}, T}^{(k)}$. 
Proof. Let $S^{\prime}=S \cup\left\{s_{0}\right\}$ where $s_{0} \notin A \cup B \cup S$ and

$$
\begin{aligned}
p^{\prime}\left(s, u, v, s^{\prime}\right) & =p\left(s, u, v, s^{\prime}\right) \quad \text { if } s, s^{\prime} \in S, u \in A \cup B, v \in A \cup B \cup S \cup\{+,-, \cdot\}, \\
& =h\left(s^{\prime}\right) \quad \text { if } s=s_{0}, s^{\prime} \in S, u=v \in A \cup B \\
h^{\prime}(s) & =1 \quad \text { if } s=s_{0}, \\
& =0 \quad \text { if } s \neq s_{0} .
\end{aligned}
$$

Clearly, $Z^{\prime}$ has the desired properties.

By virtue of the above theorem, we may, without loss of generality, consider only those PTM with an initial state. If $h$ is concentrated at $s$, we shall also write $Z=(A, B, S, p, s)$ for $Z=(A, B, S, p, h)$.

IV. Relative computability. In this section, $A$ will denote the ordinary finite set $\left\{a_{1}, a_{2}, \ldots, a_{m}\right\}$ where $m>0$ and $a_{1}=1$. Moreover, $T$ will denote a random set in $A^{*}$.

Definition 4.1. A $k$-ary random function $f$ in $A^{*}$ is partially $T$-computable iff, for some PTM $Z=(A, B, S, p, h), f=\Phi_{Z, T}^{(k)}$. In this case, we say that $Z T$-computes $f$. If, in addition, $f$ is total, then $f$ is $T$-computable.

REMARK. In the above definition, if $T$ is a crisp subset and $Z$ is deterministic, then $f$ is a crisp function. In this case, we say that $f$ is partially $T$-recursive. It is easily seen that the concept of partially $T$-recursive as given here is equivalent to the existing one [3]. If $f$ is total, then we say that $f$ is $T$-recursive.

Definition 4.2. A $k$-ary random function $f$ in $A^{*}$ is partially computable iff, for some simple $\operatorname{PTM} Z=(A, B, S, p, h), f=\Phi_{Z}^{(k)}$. In this case, we say that $Z$ computes $f$. If, in addition, $f$ is total, then $f$ is computable.

THEOREM 4.1. If a $k$-ary random function is (partially) computable, then it is (partially) $T$-computable for every random set $T$ in $A^{*}$.

Proof. Immediate from the definitions.

THEOREM 4.2. Let $f$ be a $k$-ary random function in $A^{*} . f$ is (partially) computable iff $f$ is (partially) $\varnothing$-computable, where $\varnothing$ is the empty set.

Proof. If $f$ is (partially) computable, then by Theorem $4.1, f$ is (partially) $\varnothing$ computable. Conversely, if $f$ is (partially) $\varnothing$-computable, then there exists a PTM $Z=(A, B, S, p, h)$ which $\varnothing$-computes $f$. Let $Z^{\prime}=\left(A, B, S, p^{\prime}, h\right)$ where

$$
\begin{aligned}
p^{\prime}\left(s, u, v, s^{\prime}\right) & =p\left(s, u, v, s^{\prime}\right) & & \text { if } v \notin S \text { and } u \neq v, \\
& =p\left(s, u, u, s^{\prime}\right)+\sum_{s^{\prime \prime} \in S} p\left(s, u, s^{\prime \prime}, s^{\prime}\right) & & \text { if } u=v .
\end{aligned}
$$

Then $Z^{\prime}$ is a simple PTM and computes $f$.

By virtue of the above theorem, for each statement which is true for $T$ computability, a corresponding statement which is true for computability may be obtained simply by taking $T=\varnothing$. 
Definition 4.3. The characteristic function of a $k$-ary random set $C$ in $A^{*}$ is the $k$-ary random function $\chi_{C}$ where

$$
\begin{aligned}
\mu_{x_{C}}\left(w^{(k)}, w\right) & =\mu_{C}\left(w^{(k)}\right) & & \text { if } w=1, \\
& =1-\mu_{C}\left(w^{(k)}\right) & & \text { if } w=e, \\
& =0 & & \text { otherwise. }
\end{aligned}
$$

The characteristic function of a $k$-ary random predicate in $A^{*}$ is the characteristic function of its extension.

COROLLARY 4.1. Let $\chi_{P}$ be the characteristic function of a $k$-ary random predicate $P$ in $A^{*}$, then

$$
\begin{aligned}
\mu_{x_{P}}\left(w^{(k)}, w\right) & =\mu_{P}\left(w^{(k)}\right) & & \text { if } w=1, \\
& =1-\mu_{P}\left(w^{(k)}\right) & & \text { if } w=e, \\
& =0 & & \text { otherwise. }
\end{aligned}
$$

Definition 4.4. A $k$-ary random set $C$ in $A^{*}$ is $T$-computable iff $\chi_{C}$ is $T$ computable. A $k$-ary random predicate $P$ in $A^{*}$ is $T$-computable iff $E_{P}$ is $T$ computable.

THEOREM 4.3. $T$ is T-computable.

Proof. Let $Z=\left(A, B, S, p, s_{0}\right)$ where $B=\{*, b\}, S=\left\{s_{0}, s_{1}, s_{2}, s_{3}, s_{4}\right\}$ and

$$
\begin{aligned}
p\left(s_{0}, a, s_{1}, s_{2}\right) & =1 \quad \text { for all } a \in A \cup B, \\
p\left(s_{1}, a, 1, s_{3}\right) & =1 \quad \text { for all } a \in A \cup B, \\
p\left(s_{3}, 1, R, s_{2}\right) & =1, \\
p\left(s_{2}, a, b, s_{4}\right) & =1 \quad \text { for all } a \in A, \\
p\left(s_{4}, b, R, s_{2}\right) & =1 .
\end{aligned}
$$

Then $\chi_{T}=\Phi_{z, T}$.

Operations of $T$-computable random functions, sets and predicates will be considered next. It will be assumed that all symbols mentioned below are distinct unless otherwise stated. Moreover, if $Z_{i}=\left(A, B_{i}, S_{i}, p_{i}, h_{i}\right), i=1,2$, are PTM's, then

1. $B_{i} \cap S_{j}=\varnothing, i, j=1,2$,

2. $B_{1} \cap B_{2}=\{*, b\}$ unless otherwise stated,

3. unless explicitly mentioned, no elements will be assumed to belong to $S_{1} \cap S_{2}$.

Definition 4.5. Let $Z_{i}=\left(A, B_{i}, S_{i}, p_{i}, h_{i}\right), i=1,2$, be PTM and $S_{3} \subseteq S_{1} \cap S_{2} \neq \varnothing$. By $Z_{1} \rightarrow Z_{2}\left(\bmod S_{3}\right)$ we mean the PTM $Z=(A, B, S, p, h)$ where $B=B_{1} \cup B_{2}$, $S=S_{1} \cup S_{2}$ and

$$
\begin{aligned}
p\left(s, u, v, s^{\prime}\right) & =p_{1}\left(s, u, v, s^{\prime}\right) & & \text { if } s \in\left(S_{1}-S_{2}\right) \cup S_{3}, u \in U_{1}, v \in V_{1}, s^{\prime} \in S_{1}, \\
& =p_{2}\left(s, u, v, s^{\prime}\right) & & \text { if } s \in S_{2}-S_{3}, u \in U_{2}, v \in V_{2}, s^{\prime} \in S_{2} \\
h(s) & =h_{1}(s) & & \text { if } s \in S_{1}, \\
& =0 & & \text { otherwise. }
\end{aligned}
$$


Here, $U_{i}=A_{i} \cup B_{i}, V_{i}=U_{i} \cup S_{i} \cup\{+,-, \cdot\}, i=1$, 2. If $S_{3}=\varnothing$, we shall simply write $Z_{1} \rightarrow Z_{2}$. In general, $Z_{1} \rightarrow Z_{2} \rightarrow \cdots \rightarrow Z_{n}=\left(Z_{1} \rightarrow Z_{2} \rightarrow \cdots \rightarrow Z_{n-1}\right) \rightarrow Z_{n}$.

Definition 4.6. Let $Z=(A, B, S, p, h)$ be a PTM and $s \in S$. $s$ is a terminating state of $Z$ iff $p(s, u, \cdot s)=1$ for all $u \in A \cup B$.

LEMMA 4.1. For every $j, k, l \geqq 0$, there exists a simple deterministic PTM $Z=\left(A, B, S, p, s_{0}\right)$ such that for every $T$ and $n>0$,

$$
\begin{aligned}
& t_{z, T}\left(s_{0}\left(\overline{x^{(k)}, w^{(n)}, y^{(l)}}\right), \beta\right)=1 \quad \text { if } \beta=s\left(\overline{x^{(k)}, w^{(n)}, \ldots, w^{(n)}, y^{(l)}}\right),\left(w^{n} j \text { times }\right) \\
& =0 \text { otherwise, }
\end{aligned}
$$

where $s$ is a terminating state of $Z$.

The PTM $Z$ above will be referred to as the $[k, j, l]$-copying machine with final state $s$.

LEMMA 4.2. For every $k>0$, there exists a simple deterministic PTM $Z$ $=\left(A, B, S, p, s_{0}\right)$ such that for every $T$ and $n>0$,

$$
\begin{aligned}
t_{z, T}\left(s_{0}\left(\overline{x^{(k)}, y^{(n)}}\right), \beta\right) & =1 & & \text { if } \beta=s\left(\overline{y^{(n)}, x^{(k)}}\right), \\
& =0 & & \text { otherwise, }
\end{aligned}
$$

where $s$ is a terminating state of $Z$.

The PTM $Z$ above will be referred to as the $k$-transfer machine with final state $s$.

The existence of the PTM given in Lemmas 4.1 and 4.2 are well known $[3,13]$.

LEMMA 4.3. For every $k, l \geqq 0$ and $Z=\left(A, B, S, p, s_{0}\right)$, there exists a PTM $Z^{\prime}=\left(A, B^{\prime}, S^{\prime}, p^{\prime}, s_{1}\right)$ such that for every $T$ and $n>0$,

$$
\begin{aligned}
t_{z^{\prime}, T}\left(s_{1}\left(\overline{x^{(k)}, w^{(l)}, y^{(n)}}\right), \beta\right) & =t_{z, T}\left(s, \overline{w^{(l)}}, \gamma\right) & & \text { if } \beta=s\left(\overline{x^{(k)},\langle\gamma\rangle, y^{(n)}}\right), \\
& =0 & & \text { otherwise, }
\end{aligned}
$$

where $s$ is a terminating state of $Z$.

The proof of Lemma 4.3 is similar to that of the deterministic case $[3,13]$ and thus will be omitted. The PTM $Z^{\prime}$ given will be referred to as $[k, l]$-restriction of $Z$ with final state $s$.

Definition 4.7. Let $f$ be a $l$-ary random function in $A^{*}$ and $g_{1}, g_{2}, \ldots, g_{l}$ be $k$-ary random functions in $A^{*}$. The operation of composition associates with $f, g_{1}, g_{2}, \ldots, g_{l}$ a $k$-ary random function $h$ in $A^{*}$ where

$$
\mu_{h}\left(w^{(k)}, w\right)=\sum_{x^{(l)} \in\left(A^{*}\right)^{l}}\left\{\mu_{f}\left(x^{(l)}, w\right) \prod_{i=1}^{l} \mu_{g_{i}}\left(w^{(k)}, x_{i}\right)\right\} .
$$

We shall represent $h$ by $h\left(w^{(k)}\right)=f\left(g_{1}\left(w^{(k)}\right), g_{2}\left(w^{(k)}\right), \ldots, g_{l}\left(w^{(k)}\right)\right)$.

THEOREM 4.4. If $f, g_{1}, g_{2}, \ldots, g_{l}$ are (partially) T-computable, so is $h$ given by (4.1). 
Proof. Let $Z_{0}=\left(A, B_{0}, S_{0}, p_{0}, s_{l+1}\right) T$-compute $f$ and $Z_{i}=\left(A, B_{i}, S_{i}, p_{i}, s_{i}\right) T$ compute $g_{i}, i=1,2, \ldots, l$. Let

1. $Z_{0}^{\prime}$ be the $[0, l, 0]$-copying machine with initial state $s_{0}$ and final state $s_{1}$.

2. $Z_{i}^{\prime}$ be the $[k(i-1), k i]$-restriction of $Z_{i}$ with initial state $s_{i}$ and final state $s_{i+1}, i=1,2, \ldots, l$.

Let $Z=Z_{0}^{\prime} \rightarrow Z_{1}^{\prime} \rightarrow Z_{2}^{\prime} \rightarrow \cdots \rightarrow Z_{l}^{\prime} \rightarrow Z_{0}$. Then $Z T$-computes $h$.

THEOREM 4.5 . Let $h\left(w_{1}^{\left(k_{1}\right)}, w_{2}^{\left(k_{2}\right)}, \ldots, w_{l}^{\left(k_{l}\right)}\right)=f\left(g_{1}\left(w_{1}^{\left(k_{1}\right)}\right), g_{2}\left(w_{2}^{\left(k_{2}\right)}\right), \ldots, g_{l}\left(w_{l}^{\left(k_{l}\right)}\right)\right)$ where

$$
\mu_{h}\left(w^{(k)}, w\right)=\sum_{x^{(l)} \in\left(A^{*}\right)^{l}}\left\{\mu_{f}\left(x^{(l)}, w\right) \prod_{i=1}^{l} \mu_{g_{i}}\left(w_{i}^{\left(k_{i}\right)}, x_{i}\right)\right\}
$$

and

$$
w^{(k)}=\left(w_{1}^{\left(k_{1}\right)}, w_{2}^{\left(k_{2}\right)}, \ldots, w_{l}^{\left(k_{l}\right)}\right) .
$$

If $f, g_{1}, g_{2}, \ldots, g_{l}$ are (partially) T-computable, so is $h$.

Proof. Follows from repeated application of Theorem 4.4 and the fact that the projection functions $I_{i}^{n}\left(w^{(n)}\right)=w_{i}, i=1,2, \ldots, n$, are computable.

Definition 4.8. For every $w \in A^{*}$, define

$$
\begin{aligned}
N(w) & =\sum_{i=1}^{r} k_{i} m^{i-1} & & \text { if } w=a_{k_{r}} a_{k_{r}-1} \cdots a_{k_{1}}, r>0, \\
& =0 & & \text { if } w=e .
\end{aligned}
$$

Conversely, define $W(n)=w$ iff $N(w)=n$.

Clearly, $W(n)$ is well defined for all nonnegative $n$. For completeness sake, we define $W(n)=e$ if $n$ is negative.

The above definition provides a code for the elements of $A^{*}$. It is clear that such a code is computable in the following sense:

Lemma 4.4. There exists a simple deterministic PTM $Z=\left(A, B, S, p, s_{0}\right)$ such that for every $T$ and $w \in A^{*}$

$$
\begin{aligned}
t_{z, T}\left(s_{0} w, \beta\right) & =1 & & \text { if } \beta=s N(w), \\
& =0 & & \text { otherwise. }
\end{aligned}
$$

The PTM $Z$ above will be referred to as the coding machine with final state $s$.

Lemma 4.5. There exists a simple deterministic PTM $Z=\left(A, B, S, p, s_{0}\right)$ such that for every $T$ and nonnegative $n$,

$$
\begin{aligned}
t_{Z, T}\left(s_{0} \bar{n}, \beta\right) & =1 & & \text { if } \beta=s W(n), \\
& =0 & & \text { otherwise, }
\end{aligned}
$$

where $s$ is a terminating state of $Z$.

The PTM $Z$ above will be referred to as the decoding machine with final state $s$. 
THEOREM 4.6. If $C$ and $D$ are $T$-computable $k$-ary random sets in $A^{*}$, so are $\sim C, C \cap D$ and $C \cup D$.

Proof. From Lemmas 4.4 and 4.5 , the 2-ary functions $f_{1}\left(w_{1}, w_{2}\right)=$ $W\left[N\left(w_{1}\right)-N\left(w_{2}\right)\right]$ and $f_{2}\left(w_{1}, w_{2}\right)=W\left[N\left(w_{1}\right) \cdot N\left(w_{2}\right)\right]$ in $A^{*}$ are $T$-computable. Since $\chi_{\sim C}\left(w^{(k)}\right)=f_{1}\left(1, \chi_{C}\left(w^{(k)}\right)\right)$ and $\chi_{C \cap D}\left(w^{(k)}\right)=f_{2}\left(\chi_{C}\left(w^{(k)}\right), \chi_{D}\left(w^{(k)}\right)\right)$, therefore $\sim C$ and $C \cap D$ are $T$-computable. Moreover, $C \cup D=\sim[(\sim C) \cap(\sim D)]$, thus $C \cup D$ is also $T$-computable.

THEOREM 4.7. If $C$ and $D$ are, respectively, $T$-computable $k$-ary and l-ary random sets in $A^{*}$, so is the $(k+l)$-ary random set $C \times D$.

Proof. Let $\bar{C}$ and $\bar{D}$ be $k+l$-ary random sets in $A^{*}$ defined as follows:

$$
\begin{aligned}
& \mu_{\bar{C}}\left(x^{(k)}, y^{(l)}\right)=\mu_{C}\left(x^{(k)}\right), \\
& \mu_{\bar{D}}\left(x^{(k)}, y^{(l)}\right)=\mu_{D}\left(y^{(l)}\right) .
\end{aligned}
$$

Clearly, both $\bar{C}$ and $\bar{D}$ are $T$-computable. Since $C \times D=\bar{C} \cap \bar{D}$, by Theorem 4.6, $C \times D$ is $T$-computable.

THEOREM 4.8. If $P$ and $Q$ are T-computable random predicates in $A^{*}$, so are $\sim P$, $P \wedge Q$ and $P \vee Q$.

Proof. Follows from Corollary 2.1 and Theorem 4.6.

DEFINITION 4.9. The operation of minimalization associates with a $k+1$-ary random function $f$ in $A^{*}$ and $w_{0} \in A^{*}$ the $k$-ary random function $h$ in $A^{*}$ where

$$
\mu_{h}\left(w^{(k)}, w\right)=\mu_{f}\left(w, w^{(k)}, w_{0}\right) \prod_{x \in A_{N(w)}} \sum_{y \neq w_{0}} \mu_{f}\left(x, w^{(k)}, y\right)
$$

Here $A_{l}=\left\{w \in A^{*}: N(w)<l\right\}$. We shall represent $h$ by

$$
h\left(w^{(k)}\right)=\operatorname{Min}_{w}\left[f\left(w, w^{(k)}\right)=w_{0}\right] .
$$

Moreover, if $P$ is a $k+1$-ary random predicate in $A^{*}$, by $\operatorname{Min}_{w} P\left(w, w^{(k)}\right)$ we shall mean $\operatorname{Min}_{w}\left[\chi_{p}\left(w, w^{(k)}\right)=1\right]$.

The above definition generalizes the usual one in two ways, namely, $f$ need not be total and $w_{0}$ is arbitrary.

LEMMA 4.6. If $f$ is partially $T$-computable, so is $\operatorname{Min}_{w}\left[f\left(w, w^{(k)}\right)=e\right]$.

Proof. Let $Z=\left(A, B, S, p, s_{0}\right) T$-compute $f$ and

1. $Z_{0}=\left(A, B_{0}, S_{0}, p_{0}, s_{0}\right)$ be a simple deterministic PTM where $B_{0}=\{*, b\}$, $S_{0}=\left\{s_{0}, s, s_{1}\right\}$ and $\mathscr{P}$ consists of $\left(s_{0}, u, L, s\right)$ for all $u \in A \cup\{b\},(s, b, *, s)$, and $\left(s, *, L, s_{1}\right)$. Then

$$
\begin{aligned}
t_{z_{0}, T}\left(s_{0} \overline{w^{(k)}}, \beta\right) & =1 & & \text { if } \beta=s_{1}\left(\overline{e, w^{(k)}}\right), \\
& =0 & & \text { otherwise. }
\end{aligned}
$$

2. $Z_{1}$ be the $[0,2,0]$-copying machine with initial state $s_{1}$ and final state $s_{2}$. 
3. $Z_{2}$ be the $[0, k+1]$-restriction of $Z$ with initial state $s_{2}$ and final state $s_{3}$.

4. $Z_{3}=\left(A, B_{3}, S_{3}, p_{3}, s_{3}\right)$ be a simple deterministic PTM where $B_{3}=\{*, b\}$, $S_{3}=\left\{s_{3}, s_{4}, \ldots, s_{15}, s_{1}\right\}$ and $\mathscr{P}$ consists of $\left(s_{3}, b, R, s_{4}\right),\left(s_{4}, *, R, s_{5}\right),\left(s_{5}, u, R, s_{5}\right)$, $u \in A \cup\{b\}, \quad\left(s_{5}, *, b, s_{6}\right), \quad\left(s_{6}, b, R, s_{7}\right), \quad\left(s_{7}, u, b, s_{6}\right), u \in A \cup\{*\}, \quad\left(s_{6}, b, R, s_{8}\right)$, $\left(s_{8}, u, b, s_{6}\right), u \in A \cup\{*\}, \quad\left(s_{8}, u, L, s_{8}\right), \quad u \in A \cup\{b\}, \quad\left(s_{8}, *, b, s_{9}\right), \quad\left(s_{9}, b, R, s_{10}\right)$, $\left(s_{3}, u, b, s_{11}\right), \quad u \in A, \quad\left(s_{11}, b, R, s_{3}\right), \quad\left(s_{3}, *, b, s_{12}\right), \quad\left(s_{12}, u, R, s_{12}\right), \quad u \in A \cup\{b\}$, $\left(s_{12}, *, L, s_{13}\right), \quad\left(s_{13}, a_{i}, a_{i+1}, s_{14}\right), \quad i=1,2, \ldots, m-1, \quad\left(s_{14}, u, L, s_{14}\right), \quad u \in A$, $\left(s_{14}, b, R, s_{1}\right),\left(s_{13}, a_{m}, a_{1}, s_{15}\right),\left(s_{15}, a_{1} L, s_{13}\right),\left(s_{13}, b, a_{1}, s_{1}\right)$. Then

$$
\begin{array}{rlrl}
t_{z_{3}, T}\left(s_{0}\left(\overline{x, y, w^{(k)}}\right), \beta\right)=1 & \text { if } x=e, \beta=s_{10}\langle y\rangle, \\
=1 & \text { if } x \neq e, \beta=s_{1}\left(\overline{W[N(y)+1], w^{(k)}}\right), \\
=0 & & \text { otherwise. }
\end{array}
$$

Let $Z_{4}=Z_{0} \rightarrow Z_{1} \rightarrow Z_{2}$ and $Z^{\prime}=Z_{4} \rightarrow Z_{3}\left(\bmod \left\{s_{1}\right\}\right)$, then $Z^{\prime} T$-computes $\operatorname{Min}_{w}\left[f\left(w, w^{(k)}\right)=e\right]$.

THEOREM 4.9. If $f$ is partially T-computable, so is $\operatorname{Min}_{w}\left[f\left(w, w^{(k)}\right)=w_{0}\right]$ for arbitrary $w_{0} \in A^{*}$.

Proof. Consider the 2-ary function $g$ in $A^{*}$ where

$$
\begin{aligned}
g(x, y)=1 & \text { if } x=y, \\
=0 & \text { if } x \neq y,
\end{aligned}
$$

and let $h\left(w, w^{(k)}\right)=g\left(w_{0}, f\left(w, w^{(k)}\right)\right)$. Let $P$ be a $k+1$-ary random predicate where $\chi_{p}=h$. Then

$$
\operatorname{Min}_{w}\left[f\left(w, w^{(k)}\right)=w_{0}\right]=\operatorname{Min}_{w}\left[\chi_{\sim p}\left(w, w^{(k)}\right)=e\right] .
$$

Definition 4.10. Let $f$ and $g$ be, respectively, $k$-ary and $k+2$-ary random functions in $A^{*}$. The operation of primitive recursion associates with $f$ and $g$ the $k+1$ ary function $h$ in $A^{*}$ where $\mu_{h}$ is defined inductively on $N(x), x \in A^{*}$,

$$
\begin{aligned}
& \mu_{h}\left(e, w^{(k)}, w\right)=\mu_{f}\left(w^{(k)}, w\right), \\
& \mu_{h}\left(W[N(x)+1], w^{(k)}, w\right)=\sum_{y \in A^{*}} \mu_{h}\left(x, w^{(k)}, y\right) \cdot \mu_{g}\left(x, y, w^{(k)}, w\right) .
\end{aligned}
$$

The usual notation of primitive recursion will be used, i.e., we shall represent $h$ by

$$
\begin{aligned}
& h\left(e, w^{(k)}\right)=f\left(w^{(k)}\right), \\
& h\left(W[N(x)+1], w^{(k)}\right)=g\left(x, h\left(x, w^{(k)}\right), w^{(k)}\right) .
\end{aligned}
$$

THEOREM 4.10. If $f$ and $g$ are (partially) T-computable, so is $h$ given by (4.3).

Proof. Let $Z_{1}$ and $Z_{2} T$-compute $f$ and $g$, respectively, and

1. $Z_{0}$ be the $[0,1]$-restriction of the coding machine with initial state $s_{0}$ and final state $s_{1}$.

2. $Z_{3}$ be the $[1,2,0]$-copying machine with initial state $s_{1}$ and final state $s_{2}$. 
3. $Z_{4}$ be the $[0, k]$-restriction of $Z_{1}$ with initial state $s_{2}$ and final state $s_{3}$.

4. $Z_{5}=\left(A, B_{5}, S_{5}, p_{5}, s_{3}\right)$ be a simple deterministic PTM where $B_{5}=\{*, b\}$, $S_{5}=\left\{s_{3}, s_{4}, \ldots, s\right\}$ and $\mathscr{P}$ consists of $\left(s_{3}, b, L, s_{4}\right),\left(s_{4}, b, *, s_{4}\right),\left(s_{4}, *, L, s_{5}\right)$, $\left(s_{3}, 1, R, s_{6}\right), \quad\left(s_{6}, *, L, s_{7}\right), \quad\left(s_{7}, 1, b, s_{7}\right), \quad\left(s_{7}, b, L, s_{8}\right), \quad\left(s_{8}, b, *, s_{8}\right), \quad\left(s_{8}, *, L, s_{9}\right)$, $\left(s_{9}, b, 1, s_{5}\right),\left(s_{6}, 1, R, s_{6}\right),\left(s_{6}, *, L, s_{10}\right),\left(s_{10}, 1, b, s_{10}\right),\left(s_{10}, b, L, s_{11}\right),\left(s_{11}, 1, *, s_{12}\right)$, $\left(s_{12}, *, L, s_{12}\right),\left(s_{12}, 1, L, s_{12}\right),\left(s_{12}, b, 1, s_{13}\right),\left(s_{13}, 1, L, s_{13}\right),\left(s_{13}, b, 1, s_{5}\right)$. Then

$$
\begin{aligned}
t_{Z_{5}, T}\left(s_{3} n * \alpha, \beta\right) & =1 & & \text { if } \beta=s_{5} \bar{n}^{*} b^{*} \alpha, \\
& =0 & & \text { otherwise, }
\end{aligned}
$$

for all $\alpha \in\left(A \cup B_{5}\right)^{*}$.

5. $Z_{6}=\left(A, B_{6}, S_{6}, p_{6}, s_{14}\right)$ be a simple deterministic PTM where $B_{6}=\{*, b\}$, $S_{6}=\left\{s_{14}, s_{15}, \ldots, s_{28}\right\}$ and $\mathscr{P}$ consists of $\left(s_{14}, b, R, s_{14}\right),\left(s_{14}, *, b, s_{15}\right),\left(s_{15}, u, R, s_{15}\right)$, $u \in A \cup\{b\},\left(s_{15}, *, R, s_{16}\right),\left(s_{16}, u, R, s_{16}\right), u \in A \cup\{b\},\left(s_{16}, *, b, s_{17}\right),\left(s_{17}, b, R, s_{18}\right)$, $\left(s_{18}, u, b, s_{17}\right), u \in A \cup\{b\},\left(s_{18}, b, R, s_{19}\right),\left(s_{19}, u, b, s_{17}\right), u \in A \cup\{b\},\left(s_{19}, u, L, s_{19}\right)$, $u \in A \cup\{b\}, \quad\left(s_{19}, *, b, s_{20}\right), \quad\left(s_{20}, b, R, s_{21}\right), \quad\left(s_{17}, 1, R, s_{17}\right), \quad\left(s_{17}, *, R, s_{22}\right)$, $\left(s_{22}, b, 1, s_{23}\right), \quad\left(s_{23}, 1, L, s_{23}\right), \quad\left(s_{23}, *, L, s_{23}\right), \quad\left(s_{23}, b, R, s_{24}\right), \quad\left(s_{24}, 1, b, s_{24}\right)$, $\left(s_{24}, b, R, s_{25}\right), \quad\left(s_{22}, 1, L, s_{26}\right), \quad\left(s_{26}, *, 1, s_{26}\right), \quad\left(s_{26}, 1, L, s_{27}\right), \quad\left(s_{27}, 1, *, s_{27}\right)$, $\left(s_{27}, *, L, s_{28}\right),\left(s_{28}, 1, L, s_{28}\right),\left(s_{28}, b, R, s_{25}\right)$. Then

$$
\begin{aligned}
t_{z_{6}, T}\left(s_{14}\left(\overline{\left.\bar{n}_{1}, \bar{n}_{2}, w, w^{(k)}\right)}, \beta\right)=1\right. & \text { if } n_{1} \neq 0, \beta=s_{21} \bar{w} \\
=1 & \text { if } \left.n_{1} \neq 0, \beta=s_{25} \overline{\overline{\left(n_{1}-1\right.}}, \overline{n_{2}+1}, w, w^{(k)}\right), \\
=0 & \text { otherwise, }
\end{aligned}
$$

for every nonnegative $n_{1}$ and $n_{2}$.

6. $Z_{7}$ be the $[1,1, k+1]$-copying machine with initial state $s_{25}$ and final state $s_{29}$.

7. $Z_{8}$ be the $[2,1]$-restriction of the decoding machine with initial state $s_{29}$ and final state $s_{30}$.

8. $Z_{9}$ be the $[4,1,0]$-copying machine with initial state $s_{30}$ and final state $s_{14}$.

Let $Z_{10}=Z_{0} \rightarrow Z_{3} \rightarrow Z_{4} \rightarrow Z_{5} \rightarrow Z_{6} \rightarrow Z_{7} \rightarrow Z_{8}$ and $Z=Z_{10} \rightarrow Z_{9}\left(\bmod \left\{s_{14}\right\}\right)$, then $Z T$-computes $h$.

V. Crisp functions computable by PTM's. Let $T$ be a crisp set. From the remark made after Definition 4.1 , it is clear that every partially $T$-recursive function is a partially $T$-computable crisp function. Conversely, since one may treat a PTM as if it were just a nondeterministic Turing machine, a deterministic Turing machine may be set up which simultaneously follows all possible paths which the PTM might take. Under the hypothesis that all computation lead to the same output, it is safe to take as output the result obtained when any path reaches a halt state. This shows that every partially $T$-computable crisp function is $T$-recursive. Thus, in this sense, we gain nothing by considering PTM's. However, we shall show in this section that, in some other sense, we do gain something by considering PTM's.

Various other classes of crisp functions characterizable by PTM's will be studied 
in this section and their relationship investigated. The symbol $A$ will stand for an ordinary finite set and $T$ a crisp subset of $A^{*}$. The symbols $k$ and $l$ will stand for positive integers and $\lambda$, with or without subscripts, real number where $0 \leqq \lambda<1$.

Definition 5.1. The class $\mathscr{F}(A, T, k, \lambda)$ is the collection of all $k$-ary crisp functions in $A^{*}$ such that $m(f, g)>\lambda$ for some partially $T$-computable $k$-ary random function $g$ in $A^{*}$. Moreover,

$$
\mathscr{F}(A, T, k)=\bigcup_{0 \leqq \lambda<1} \mathscr{F}(A, T, k, \lambda) .
$$

If no ambiguity is likely to arise, we shall suppress the symbols $A, T, k$ or any combination of them.

Recall that $m(f, g)>\lambda$ means that the probability that $f\left(w^{(k)}\right)=g\left(w^{(k)}\right)$ for every $w^{(k)}$ is larger than $\lambda$ (cf. Definition 2.8).

THEOREM 5.1. $\mathscr{F}\left(\lambda_{1}\right)=\mathscr{F}\left(\lambda_{2}\right)$ for every $0 \leqq \lambda_{1}, \lambda_{2}<1$.

Proof. Without loss of generality, let $\lambda_{1}<\lambda_{2}$. It follows immediately from the above definition that $\mathscr{F}\left(\lambda_{2}\right) \subseteq \mathscr{F}\left(\lambda_{1}\right)$. Conversely, let $f \in \mathscr{F}\left(\lambda_{1}\right)$. Then

$$
m(f, g)=\prod_{w^{(k)} \in\left(A^{*}\right)^{k}} \mu_{g}\left(w^{(k)}, f\left(w^{(k)}\right)\right)>\lambda_{1}
$$

for some partially $T$-computable $k$-ary random function $g$ in $A^{*}$. Therefore, there exists a finite subset $\boldsymbol{X}$ of $\left(A^{*}\right)^{k}$ such that

$$
\prod_{\omega^{(k)} \in X^{\prime}} \mu_{g}\left(w^{(k)}, f\left(w^{(k)}\right)\right)>\lambda_{2}
$$

where $\boldsymbol{X}^{\prime}=\left(A^{*}\right)^{k}-\boldsymbol{X}$. Let $\boldsymbol{g}^{\prime}$ be a $k$-ary random function in $A^{*}$ defined as follows:

$$
\begin{aligned}
\mu_{g^{\prime}}\left(w^{(k)}, w\right) & =\mu_{g}\left(w^{(k)}, w\right) & & \text { if } w^{(k)} \in \boldsymbol{X}^{\prime}, \\
& =1 & & \text { if } w^{(k)} \in \boldsymbol{X} \text { and } w=f\left(w^{(k)}\right), \\
& =0 & & \text { otherwise. }
\end{aligned}
$$

Clearly, $g^{\prime}$ is partially $T$-computable and $m\left(f, g^{\prime}\right)>\lambda_{2}$. Thus $f \in \mathscr{F}\left(\lambda_{2}\right)$.

CoROllaRy 5.1. $\mathscr{F}=\mathscr{F}(\lambda)$ for all $0 \leqq \lambda<1$.

THEOREM 5.2. If $f \in \mathscr{F}(l), g_{i} \in \mathscr{F}(k), i=1,2, \ldots, l$, then

$$
h\left(w^{(k)}\right)=f\left(g_{1}\left(w^{(k)}\right), \ldots, g_{l}\left(w^{(k)}\right)\right) \in \mathscr{F}(k) .
$$

Proof. Let $m\left(f, f^{\prime}\right)>\lambda$ and $m\left(g_{i}, g_{i}^{\prime}\right)>\lambda, i=1,2, \ldots, l$, where $f^{\prime}$ and $g_{i}^{\prime}, i=1,2$, $\ldots, l$, are partially $T$-computable. Let $h^{\prime}\left(w^{(k)}\right)=f^{\prime}\left(g_{1}\left(w^{(k)}\right), \ldots, g_{l}\left(w^{(k)}\right)\right)$. Then $m\left(h, h^{\prime}\right)>\lambda^{l}$.

DEFINITION 5.2. Let $p$ be a real number where $0 \leqq p \leqq 1$. The 1 -ary crisp functions $F_{p}$ and $G_{p}$ in $\{1\}^{*}$ are defined as follows:

$$
F_{p}(n)=\sum_{i=0}^{n} 2^{i} \delta_{n+1-i}, \quad G_{p}(n)=\delta_{n+1},
$$


where $\cdot \delta_{1} \delta_{2} \cdots \delta_{n} \cdots$ is the binary expansion of $p$. Here, we have used interchangeably the word $\bar{n}$ and the nonnegative integer $n$. The same convention will be used throughout the rest of the paper.

Definition 5.3. Let $p$ be a real number where $0 \leqq p \leqq 1 . p$ is said to be computable iff $F_{p}$, or equivalently $G_{p}$, is recursive. Otherwise, $p$ is noncomputable.

Properties of computable real numbers can be found elsewhere [7]. It is well known that there exist noncomputable real numbers. However,

THEOREM 5.3. $F_{p} \in \mathscr{F}$ for every $0 \leqq p \leqq 1$.

Proof. Let $Z=\left(A, B, S, p, s_{0}\right)$ be a simple PTM where $A=\{1\}, B=\{*, b\}$, $S=\left\{s_{0}, s_{1}\right\}$ and

$$
\begin{aligned}
& p\left(s_{0}, 1,1, s_{1}\right)=p, \quad p\left(s_{0}, 1, b, s_{1}\right)=1-p, \\
& p\left(s_{1}, 1, R, s_{0}\right)=1, \quad p\left(s_{1}, b, R, s_{0}\right)=1 .
\end{aligned}
$$

Let $g_{1}=\Phi_{z}$. Then

$$
\mu_{g_{1}}(n, l)=\left(\begin{array}{c}
n \\
l
\end{array}\right) p^{l}(1-p)^{n-l}
$$

where $\left(\begin{array}{l}n \\ l\end{array}\right)$ is the binomial coefficient and $\left(\begin{array}{l}n \\ l\end{array}\right)=0$ if $n<l$. Let

$$
g_{2}(n)=4^{n+1}, \quad g_{3}(n, l)=\sum_{i=0}^{n} 2^{i} \varepsilon_{n+1-i},
$$

where $\varepsilon_{1} \varepsilon_{2} \cdots \varepsilon_{n} \cdots$ is the binary expansion of $l / 4^{n+1}$. Let

$$
g(n)=g_{3}\left\{n, g_{1}\left[g_{2}(n)\right]\right\} \text {. }
$$

Since $g_{1}, g_{2}$ and $g_{3}$ are computable, so is $g$. We shall show that $m(f, g)>0$. To this end, consider the collection $J$ of all infinite sequences of 0 's and 1's. Let $J\left(\rho_{1}, \rho_{2}, \ldots, \rho_{n}\right)$ be the subset of $J$ consisting of all sequences starting with $\left(\rho_{1}, \rho_{2}, \ldots, \rho_{n}\right) . J$ shall be considered to be a measurable space [5] whose measurable sets are the $\sigma$-ring [5] generated by the sets $J\left(\rho_{1}, \rho_{2}, \ldots, \rho_{n}\right)$. To each $J\left(\rho_{1}, \rho_{2}, \ldots, \rho_{n}\right)$ we assign a measure $\left(\begin{array}{l}n \\ l\end{array}\right) p^{l}(1-p)^{n-l}$ where $l$ is the number of 1 's in $\left(\rho_{1}, \rho_{2}, \ldots, \rho_{n}\right)$. This induces a probability measure $m_{p}$ on $J$. From the definition of $g$, we have

$$
\mu_{g}(n, f(n))=m_{p}\left\{\left|l / 4^{n+1}-p\right| \leqq 1 / 2^{n+1}\right\} .
$$

Here, we identified with each sequence $\left(\rho_{1}, \rho_{2}, \ldots, \rho_{n}, \ldots\right) \in J$ the real number whose binary expansion is $\cdot \rho_{1} \rho_{2} \cdots \rho_{n} \cdots$. By the Law of Large Numbers [12, p. 209],

$$
m_{p}\left\{\left|l / 4^{n+1}-p\right| \leqq 1 / 2^{n+1}\right\} \geqq 1-1 / 4 \cdot 4^{n+1} \cdot 4^{n+1}=1-1 / 4^{2 n+3} .
$$

Since $\prod_{n=2}^{\infty}\left(1-1 / n^{2}\right)=\frac{1}{2}>0$, therefore $\prod_{n=0}^{\infty}\left(1-1 / 4^{2 n+3}\right)>0$. Thus

$$
m(f, g)=\prod_{n=0}^{\infty} \mu_{g}(n, f(n))>0 .
$$


THEOREM 5.4. $G_{p} \in \mathscr{F}$ for every $0 \leqq p \leqq 1$.

Proof. Let $f(n)=\delta$ where $n \equiv \delta(\bmod 2), \delta \in\{0,1\}$. Then $G_{p}(n)=f\left(F_{p}(n)\right)$. Since $f$ is computable, by Theorems 5.3 and $5.2, G_{p} \in \mathscr{F}$.

The following well-known result [3] will be needed in later discussions. It will be quoted below without proof.

THEOREM 5.5. Let $I$ be the set of nonnegative integers and $k>0$. There exist a recursive $k$-ary function $K$ in $I$ and $k$ recursive 1 -ary functions $L_{1}, L_{2}, \ldots, L_{k}$, in I such that

$$
\begin{gathered}
K\left(L_{1}(x), L_{2}(x), \ldots, L_{k}(x)\right)=x \text { for all } x \in I, \\
L_{i}\left(K\left(x_{1}, x_{2}, \ldots, x_{k}\right)\right)=x_{i} \text { for all } x_{1}, x_{2}, \ldots, x_{k} \in I \text { and } i=1,2, \ldots, k .
\end{gathered}
$$

Combining the above theorem with Lemmas 4.4 and 4.5 yields

THEOREM 5.6. For every $k>0$, there exist a recursive $k$-ary function $K$ in $A^{*}$ and $k$ recursive 1-ary functions $L_{1}, L_{2}, \ldots, L_{k}$ in $A^{*}$ such that

$$
\begin{gathered}
K\left(L_{1}(w), L_{2}(w), \ldots, L_{k}(w)\right)=w \text { for all } w \in I, \\
L_{i}\left(K\left(w^{(k)}\right)\right)=w_{i} \text { for all } w^{(k)} \in\left(A^{*}\right)^{k} \text { and } i=1,2, \ldots, k .
\end{gathered}
$$

To simplify. our notation, we shall write

and

$$
N_{k}\left(w^{(k)}\right)=N\left(K\left(w^{(k)}\right)\right)
$$

Clearly,

$$
W_{k}(n)=\left(L_{1}(W(n)), L_{2}(W(n)), \ldots, L_{k}(W(n))\right)
$$

$$
W_{k}\left[N_{k}\left(w^{(k)}\right)\right]=w^{(k)} \quad \text { and } \quad N_{k}\left[W_{k}(n)\right]=n .
$$

THEOREM 5.7. For every $A, T$, and $k, \mathscr{C}(A, T, k)$ is a proper subset of $\mathscr{F}(A, T, k)$, where $\mathscr{C}(A, T, k)$ is the class of all partially $T$-computable crisp functions in $A^{*}$.

Proof. Since it is obvious that $\mathscr{C}(A, T, k) \subseteq \mathscr{F}(A, T, k)$, it suffices to exhibit a $f \in \mathscr{F}(A, T, k)$ but $f \notin \mathscr{C}(A, T, k)$. Let $p \in[0,1]$ be a noncomputable real number. From Theorems 5.4, 5.3, and 5.6

$$
f\left(w^{(k)}\right)=G_{p}\left[N_{k}\left(w^{(k)}\right)\right] \in \mathscr{F}(A, T, k) .
$$

On the other hand, if $f \in \mathscr{C}(A, T, k)$, so is $G_{p}(n)=f\left[W_{k}(n)\right]$, a contradiction to the fact that $p$ is noncomputable.

Definition 5.4. The class $\mathscr{G}(A, T, k, \lambda)$ is the class of all $k$-ary crisp functions in $A^{*}$ such that $f^{\lambda} \mathrm{\sim}$ for some partially $T$-computable $k$-ary random function $g$ in $A^{*}$. Moreover,

$$
\mathscr{G}(A, T, k)=\bigcup_{0 \leqq \lambda<1} \mathscr{G}(A, T, k, \lambda) .
$$

We shall suppress $A, T, k$ or any combination of them if it is clear from the context. 
$\mathscr{G}(\{1\}, \varnothing, k, \lambda)$ is closely related to the class $\mathscr{H}(k, \lambda)$ of all probabilistically computable $k$-ary functions [9] with threshold $\lambda \geqq \frac{1}{2}$. Indeed $\mathscr{G}(\lambda) \subseteq \mathscr{H}(\lambda)$ for all $\frac{1}{2} \leqq \lambda<1$.

COROLlaRY 5.2. $\mathscr{G}\left(\lambda_{1}\right) \subseteq \mathscr{G}\left(\lambda_{2}\right)$ if $\lambda_{1} \geqq \lambda_{2}$.

COROLlaRY 5.3. $\mathscr{F} \subseteq \mathscr{G}(\lambda)$ for every $0 \leqq \lambda<1$.

THEOREM 5.8. If $f \in \mathscr{F}(k+1, \lambda)$, then $g\left(w^{(k)}\right)=\operatorname{Min}_{w}\left[f\left(w, w^{(k)}\right)=w_{0}\right] \in \mathscr{G}(k, \lambda)$ for every $w_{0} \in A^{*}$.

Proof. Let $m\left(f, f^{\prime}\right)>\lambda$ where $f^{\prime}$ is partially $T$-computable and let

$$
g^{\prime}\left(w^{(k)}\right)=\operatorname{Min}_{w}\left[f^{\prime}\left(w, w^{(k)}\right)=w_{0}\right],
$$

which is also partially $T$-computable by Theorem 4.9. From (4.2), we have, for $w=g\left(w^{(k)}\right)$,

$$
\mu_{g^{\prime}}\left(w^{(k)}, w\right) \geqq \mu_{f^{\prime}}\left(w, w^{(k)}, f\left(w, w^{(k)}\right)\right) \prod_{x \in A_{N(w)}} \mu_{f^{\prime}}\left(x, w^{(k)}, f\left(x, w^{(k)}\right)\right)
$$

since $f\left(x, w^{(k)}\right) \neq w_{0}$ for $x \in A_{N(w)}$. Thus

$$
\mu_{g^{\prime}}\left(w^{(k)}, g\left(w^{(k)}\right)\right)>\lambda
$$

for all $w^{(k)}$ or $g \stackrel{\lambda}{\sim} g^{\prime}$.

THEOREM 5.9. Let $f$ be a $k$-ary crisp function in $A^{*}$. Then $f \in \mathscr{G}(A, T, k, \lambda)$ for every $T$ and $0 \leqq \lambda<1$.

Proof. Let $g$ be a $(k+1)$-ary crisp function in $A^{*}$ defined as follows:

$$
\begin{array}{rlrl}
g\left(w^{(k)}, w\right)=1 & \text { if } f\left(w^{(k)}\right)=w, \\
& =e & \text { if } f\left(w^{(k)}\right) \neq w .
\end{array}
$$

Let $h(n)=g\left(W_{k+1}(n)\right)$. By Theorems 5.4 and 5.1, $h \in \mathscr{F}(\lambda)$. Since $g\left(w^{(k+1)}\right)$ $=h\left[N_{k+1}\left(w^{(k+1)}\right)\right]$, by Theorem 5.2, $g \in \mathscr{F}(\lambda)$. Moreover

$$
f\left(w^{(k)}\right)=\operatorname{Min}_{w}\left[g\left(w^{(k)}, w\right)=1\right] .
$$

Thus, by Theorem 5.8, $f \in \mathscr{G}(\lambda)$.

The above result is perhaps somewhat unexpected. It says that, given any $k$-ary crisp function $f$, one can find a PTM $Z$ such that the probability that $\Phi_{z}(x)=f(x)$ can be made as close to certainty as we please for all $x$.

Corollary 5.4. $\mathscr{G}=\mathscr{G}(\lambda)$ for all $0 \leqq \lambda<1$.

Corollary 5.5. $\mathscr{G}=\mathscr{H}(\lambda)$ for all $\frac{1}{2} \leqq \lambda<1$.

VI. Computable PTM's. In this section, we shall discuss a particular class of PTM's, the computable PTM's. A necessary and sufficient condition for a $k$-ary crisp function to be partially recursive is established via computable PTM's.

Definition 6.1. A quasi PTM is a quintuple $(A, B, S, p, h)$ where $A, B, S, p$ and $h$ are the same as that given in Definition 3.1 except $p$ and $h$ need not satisfy the two conditions stipulated there. 
Given a quasi PTM $Z=(A, B, S, p, h)$ and random subsets $T_{1}$ and $T_{2}$ in $A^{*}$, define $q_{z, T_{1}, T_{2}}(\alpha, \beta)$ in a manner similar to $q_{z, T}(\alpha, \beta)$ of PTM except when $\alpha=\gamma s u \delta$, $\beta=\gamma s^{\prime} u \delta$. In this case, define

$$
\begin{aligned}
q_{z, T_{1}, T_{2}}(\alpha, \beta)=p\left(s, u, u, s^{\prime}\right) & +\sum_{s^{\prime \prime} \in S} p\left(s, u, s^{\prime}, s^{\prime \prime}\right) \mu_{T_{1}}(\langle\alpha\rangle) \\
& +\sum_{s^{\prime \prime} \in S} p\left(s, u, s^{\prime \prime}, s^{\prime}\right) \mu_{T_{2}}(\langle\alpha\rangle) .
\end{aligned}
$$

In other words, $T_{2}$ plays the role of $\sim T$. Moreover, define $q_{Z, T_{1}, T_{2}}^{(n)}$ and $t_{Z, T_{1}, T_{2}}^{(n)}$ in a manner similar to $q_{z, T}^{(n)}$ and $t_{z, T}^{(n)}$ of PTM's.

Definition 6.2. A real number $p$ is admissible iff $p=\sum_{i=1}^{n} 2^{-i} \varepsilon_{i}, \varepsilon_{i} \in\{0,1\}$ for $i=1,2, \ldots, n$. In this case, define $F(p)=F_{p}(n-1)$ if $\varepsilon_{n} \neq 0$ and $F(0)=0$. The inverse of $F$ will be denoted by $F^{-1}$.

Definition 6.3. A quasi PTM $Z=(A, B, S, p, h)$ is admissible iff the range of $p$ and $h$ is a subset of the set of admissible real numbers. A random subset $T$ in $A^{*}$ is admissible iff $\mu_{T}(w)$ is admissible for all $w \in A^{*}$.

Corollary 6.1. If $Z$ is an admissible quasi PTM and $T_{1}, T_{2}$ admissible random subsets, then $q_{z, T_{1}, T_{2}}(\alpha, \beta), q_{Z, T_{1}, T_{2}}^{(n)}(\alpha, \beta)$ and $t_{Z, T_{1}, T_{2}}^{(n)}(\alpha, \beta)$ are admissible for all $\alpha$ and $\beta$.

THEOREM 6.1. For every mutually disjoint finite nonempty sets. $A, B$ and $S$, the function

$$
H\left(Z, T_{1}, T_{2}, x, y^{(k)}, w\right)=F\left[t_{Z, T_{1}, T_{2}}^{(N(x)+1)}\left(y^{(k)}, w\right)\right]
$$

is recursive. Here, $Z=(A, B, S, p, h)$ is an admissible quasi PTM and $T_{1}, T_{2}$ are admissible random subsets in $A^{*}$. In (6.1), $Z, T_{1}$ and $T_{2}$ stands for $W\left[F\left(p\left(s, u, v, s^{\prime}\right)\right)\right]$, $W[F(h(s))], W\left[F\left(\mu_{T_{i}}(w)\right)\right], i=1,2$, in some fixed order.

Proof. There are only finitely many paths of $Z$ for $y^{(k)}$ of length $N(x)+1$. Moreover, the probability of each path can be found by adding and multiplying the admissible real numbers $p\left(s, u, v, s^{\prime}\right), h(s), \mu_{T_{1}}(w)$ and $\mu_{T_{2}}(w)$ finitely many times.

Definition 6.4. A PTM $Z=(A, B, S, p, h)$ is computable iff the range of $p$ and $h$ is a subset of the set of computable real numbers.

THEOREM 6.2. Let $f$ be a $k$-ary crisp function in $A^{*}$. There exist a computable PTM $Z=(A, B, S, p, h)$, random subsets $T$ in $A^{*}$ and a computable real number $\lambda \geqq \frac{1}{2}$ such that

$$
\mu_{\Phi_{Z, T}^{(k)}}\left(w^{(k)}, f\left(w^{(k)}\right)\right)>\lambda
$$

for all $w^{(k)}$ except possibly for those $w^{(k)}$ where $f\left(w^{(k)}\right)=\Omega$ iff $f$ is partially recursive.

Proof. For $n=1,2, \ldots$, let $Z_{n}=\left(A, B, S, p_{n}, h_{n}\right)$ be a PTM where

$$
\begin{aligned}
p_{n}\left(s, u, v, s^{\prime}\right) & =F^{-1}\left[F_{p\left(s, u, v, s^{\prime}\right)}(n-1)\right], \\
h_{n}(s) & =F^{-1}\left[F_{h(s)}(n-1)\right],
\end{aligned}
$$


and let $T_{n}^{\prime}$ and $T_{n}^{\prime \prime}$ be random subsets in $A^{*}$ where

$$
\mu_{T_{n}^{\prime}}(w)=F^{-1}\left[F_{\mu_{T}(w)}(n-1)\right], \quad \mu_{T_{n}^{n}}(w)=F^{-1}\left[F_{\mu_{T}(w)}(n-1)\right] .
$$

Let

$$
\begin{aligned}
g_{1}(x, y)=1 & \text { if } N(x)>N(y) \\
=e & \text { if } N(x) \leqq N(y)
\end{aligned}
$$

and

$$
g_{2}\left(x, y^{(k)}, w\right)=g_{1}\left[F_{\lambda}(n-1)+1, H\left(Z_{n}, T_{n}^{\prime}, T_{n}^{\prime \prime}, n, y^{(k)}, w\right)\right]
$$

where $n=N(x)$. Then

$$
f\left(y^{(k)}\right)=L_{2}\left\{\operatorname{Min}_{w}\left[g_{2}\left(L_{1}(w), y^{(k)}, L_{2}(w)\right)=1\right]\right\} .
$$

Since all the functions involved are recursive, thus $f$ is partially recursive. The converse is trivial.

ACKnowledgement. The author is indebted to the referee for pointing out an error in the proof of one of the theorems.

\section{REFERENCES}

1. J. W. Carlyle, Reduced forms for stochastic sequential machines, J. Math. Anal. Appl. 7 (1963), 167-175. MR 31 \#4695.

2. K. L. Chung, Markov chains with stationary transition probabilities, Die Grundlehren der math. Wissenschaften, Band 104, Springer-Verlag, Berlin, 1960. MR 22 \#7176.

3. M. Davis, Computability and unsolvability, McGraw-Hill Series in Information Processing and Computers, McGraw-Hill, New York, 1958. MR 23 \#A1525.

4. W. Feller, An introduction to probability theory and its applications. Vol. 1, Wiley, New York, 1950. MR 12, 424.

5. P. R. Halmos, Measure theory, Van Nostrand, Princeton, N. J., 1950. MR 11, 504.

6. K. de Leeuw, E. F. Moore, C. E. Shannon and N. Shapiro, Computability by probabilistic machines, Automata Studies, Ann of Math. Studies, no. 34, Princeton Univ. Press, Princeton, N. J., 1956, pp. 183-212. MR 18, 104.

7. M. Minsky, Computation: Finite and infinite machines, Prentice-Hall, Englewood Cliffs, N. J., 1967.

8. M. O. Rabin, Probabilistic automata, Information and Control 6 (1963), 230-245.

9. E. S. Santos, Probabilistic Turing machines and computability, Proc. Amer. Math. Soc. 22 (1969), 704-710. MR 40 \#2468.

10. D. Scott, Some definitional suggestions for automata theory, J. Comput. System Sci. 1 (1967), 187-212.

11. A. M. Turing, On computable numbers, with an application to the entscheidungs problem, Proc. London Math. Soc. (2) 42 (1936), 230-265.

12. J. V. Uspensky, Introduction to mathematical probability, McGraw-Hill, New York, 1937.

13. V. Vukovic, Basic theorems on Turing algorithms, Publ. Inst. Math. 1 (15) (1961), 31-65.

14. L. A. Zadeh, Fuzzy sets, Information and Control 8 (1965), 338-353. MR 36 \#2509.

Youngstown State University,

Youngstown, OHIO 44503 